Cahiers $d u$ MONDE RUSSE

\section{Cahiers du monde russe}

Russie - Empire russe - Union soviétique et États indépendants

45/3-4 | 2004

Varia

\title{
Meyerhold et le symbolisme
}

\author{
GÉrard Abensour
}

\section{OpenEdition \\ Journals}

Édition électronique

URL : https://journals.openedition.org/monderusse/8705

DOI : $10.4000 /$ monderusse. 8705

ISSN : $1777-5388$

\section{Éditeur}

Éditions de l'EHESS

\section{Édition imprimée}

Date de publication : 1 juillet 2004

Pagination : 591-606

ISBN : 2-7132-2009-2

ISSN : $1252-6576$

\section{Référence électronique}

GÉrard Abensour, " Meyerhold et le symbolisme », Cahiers du monde russe [En ligne], 45/3-4 | 2004, mis en ligne le 01 janvier 2007, consulté le 02 septembre 2022. URL : http://journals.openedition.org/ monderusse/8705; DOI : https://doi.org/10.4000/monderusse.8705 
chercher : repérer : avancer

Cet article est disponible en ligne à l'adresse :

http://www.cairn.info/article.php?ID REVUE=CMR\&ID NUMPUBLIE=CMR 453\&ID ARTICLE=CMR 453 0591

\title{
Meyerhold et le symbolisme
}

\author{
par GÉRARD ABENSOUR
}

\section{Editions de I'EHESS | Cahiers du monde russe}

\author{
2004/3-4 - Vol 45 \\ ISSN 1252-6576 | ISBN 2713220092 | pages 591 à 606
}

Pour citer cet article :

—ABENSOUR G., Meyerhold et le symbolisme, Cahiers du monde russe 2004/ 3-4, Vol 45, p. 591-606.

Distribution électronique Cairn pour les Editions de l'EHESS.

() Editions de l'EHESS. Tous droits réservés pour tous pays.

La reproduction ou représentation de cet article, notamment par photocopie, n'est autorisée que dans les limites des conditions générales d'utilisation du site ou, le cas échéant, des conditions générales de la licence souscrite par votre établissement. Toute autre reproduction ou représentation, en tout ou partie, sous quelque forme et de quelque manière que ce soit, est interdite sauf accord préalable et écrit de l'éditeur, en dehors des cas prévus par la législation en vigueur en France. Il est précisé que son stockage dans une base de données est également interdit. 


\section{GÉRARD ABENSOUR}

\section{MEYERHOLD ET LE SYMBOLISME}

Parler des rapports entre Meyerhold et le symbolisme c'est évoquer d'abord le problème général de la dynamique des rapports entre le texte écrit et sa représentation scénique. C'est ensuite aborder la situation spécifique de l'esthétique symboliste par rapport au théâtre.

Réfléchissant à l'évolution de l'art moderne et en particulier du théâtre au cours de la première décennie du XX $x^{\mathrm{e}}$ siècle, Meyerhold écrit :

J'ai lu quelque part que la scène crée la littérature. La réalité est tout autre. L'influence de la scène sur la littérature, c'est uniquement d'en retarder un peu le développement [...]. Le nouveau théâtre naît de la littérature. Dans la rupture avec les anciennes formes dramatiques, c'est toujours à la littérature qu'est revenue l'initiative. Tchékhov a écrit La Mouette avant l'apparition du Théâtre artistique $^{1}$ qui l'a représentée sur scène. [...] Maeterlinck a produit ses œuvres avant la naissance de son théâtre. La littérature crée le théâtre. Et pas uniquement les auteurs dramatiques à l'origine des formes nouvelles dont ils offrent les modèles, mais aussi les critiques avec leur rejet des formes anciennes².

Le metteur en scène reconnaît donc la primauté de l'auteur sur le concepteur de la réalisation scénique.

NDLR : les noms propres russes sont translittérés selon les normes internationales, en usage dans la revue, à l'exception de Meyerhold pour lequel on a préféré conserver la transcription française de type courant.

1. On préfèrera l'expression « Théâtre artistique de Moscou » qui était courante au début du siècle, à « théâtre d'Art », nom imposé à l'époque soviétique pour marquer la différence avec l'institution créée avant la révolution. Dans sa biographie de Stanislavskij, publiée par l'Arche en 1955, Nina Gourfinkel emploie l'expression «théâtre Artistique », tandis que Claudine Amiard-Chevrel, publie en 1979 aux éditions du CNRS son étude intitulée Le Théâtre artistique de Moscou (1898-1917).

2. Vs. Meyerhold, «Présages littéraires du nouveau théâtre », in Écrits sur le théâtre, trad.

B. Picon-Vallin, Lausanne, L'Âge d'Homme, vol. 1, 1973, p. 105. 
Si l'on parle maintenant du rapport qu'entretient le symbolisme avec le théâtre, on peut se demander au premier abord s'il n'y a pas entre ces deux notions une contradiction dans les termes. Ne s'agit-il pas de deux réalités antagonistes?

Que l'on se remémore ces déclarations des symbolistes français : « Le livre est la seule scène mentale nécessaire et suffisante. » Ou encore : «Il faut reprendre à la musique son bien pour féconder la rêverie du lecteur. » La matérialité de la mise en scène est une entrave à ce monde où l'individu est relié à l'esprit des sphères extraterrestres.

Or ces deux mondes antagonistes éprouvent un furieux besoin de conjonction. Cette conjonction procède d'un double mouvement, du symbolisme vers le théâtre, du théâtre vers le symbolisme. Il ne s'agit pas dans les limites de cette étude d'analyser de manière exhaustive les tropismes en jeu, qu'il suffise de proposer quelques pistes.

Pour le symbolisme, le théâtre est un temple. Il est un instrument de diffusion de ce qui aspire confusément à être une religion et qui refuse les Églises traditionnelles. La représentation théâtrale est assimilée à un spectacle liturgique, les acteurs étant des intermédiaires entre les fidèles, identifiés aux adeptes des mystères orphiques, et le dieu qui parle par la voix de l'auteur. Le théâtre est placé dans une position de subordination. Il est nié en tant que tel.

C'est malgré l'existence de l'acteur que les Symbolistes prendront place au théâtre. Dès lors ils s’ingénieront à limiter les effets de leur présence encombrante. Nous leur demanderons de se faire oublier, de se désincarner ${ }^{3}$.

Que cherche de son côté le théâtre du côté du symbolisme?

Un plaisir purement esthétique, le défi d'incarner des œuvres réputées injouables. Le théâtre, par sa nature même, est à l'affût des goûts du public, il est attentif aux modifications de sensibilité. En outre il y a, chez certains hommes de théâtre, dans toute l'Europe, la volonté de se sortir d'un spectacle purement représentatif dans lequel on ne fait qu'imiter la vie. L'acteur veut devenir un créateur à part entière, le théâtre aspire à gagner son autonomie et le symbolisme, par son aura, va lui donner ses lettres de noblesse. Devenir un temple, certes, mais tout en restant soi-même.

En fait sous le mot de symbolisme se cachent diverses visions du rapport entre l'art et la vie. Les deux directeurs de l'EEuvre, Lugné-Poë et Mauclair, se distinguent par leur projet. Pour le premier, il s'agit d'attirer les foules, pour le second, de s'adresser à une élite. Quant aux auteurs, ce n'est pas sans hésitation qu'ils entrent dans le monde du symbolisme. Certains, comme Ibsen, Hauptmann ou Čehov, lui ouvrent la voie tout en restant liés à la matérialité de la vie. En fait on parlera plutôt d'art nouveau, de sensibilité nouvelle, et un peu de symbolisme. La nouveauté se fraie un chemin avec difficulté. Ibsen est joué par Antoine dans le style naturaliste, par Paul Fort en symboliste. Quant à Čehov, il ne sait pas lui-même comment qualifier sa nouvelle pièce La Mouette (Čajka). Créée en 1896 dans le théâtre le plus conservateur qui soit, le théâtre Alexandrinski de Saint-Pétersbourg, elle n'est

3. A. Fontanias, « Réflexions au sujet du théâtre », La Société nouvelle, avril 1893. 
comprise ni par les acteurs ni par le public au grand dam de son auteur. Ce n'est que deux ans plus tard que la pièce sera créée avec succès dans un théâtre attentif aux courants nouveaux.

Ces courants sont d'ailleurs qualifiés en Russie de «décadents » plutôt que de symbolistes. « Décadent », telle est l'injure dont Arkadina gratifie son fils dans La Mouette. Les termes d'un débat fondamental sur le rôle de l'art vont être définis à la charnière des XIXe et $\mathrm{XX}^{\mathrm{e}}$ siècle, et trouveront dans les deux textes qui suivent une ligne de partage des eaux.

En 1893, Merežkovskij rédige l'essai : Des causes de la décadence et des nouveaux courants de la littérature russe contemporaine ( $O$ pričinah upadka i o novyh tečenijah sovremennoj russkoj literatury). L'auteur, écrivain et poète, place le débat sur le plan philosophique. Il se réclame de Kant. Le «noumène » (l'être en soi) ne peut être saisi par nos sens. Mais il existe et nous en avons conscience, nous ne pouvons vivre sans lui. L'homme est attiré précisément par ce qu'il ne peut connaître rationnellement, mais qui peut être suggéré par les artistes, c'est-à-dire ceux qui approfondissent les moyens d'expression relevant de chacun de nos sens. Comme exemple concret de cette perception artistique, il se réfère aux poètes maudits et en particulier à Verlaine. L'art nouveau est l'expression de l'incommunicable.

En 1897, Tolstoj publie un traité à allure de pamphlet: Qu'est-ce-que l'art ? (Čto takoe iskusstvo ?) Le grand écrivain qui se veut penseur prend le contre-pied du modernisme. Il se moque de l'art décadent et symboliste, s'ingénie à démontrer que Verlaine écrit du charabia. Pour lui, il n'y a d'art que dans la transmission aux autres de sentiments éprouvés par l'artiste. Ce qui compte, c'est la sincérité et la soumission aux grands idéaux du Bien, du Vrai, du Beau. L'art est transmission de valeurs.

\section{II}

Meyerhold est entré comme acteur au Théâtre artistique de Moscou dès sa création en 1898. Il y crée le rôle de Treplev, le personnage de La Mouette qui incarne le mal de vivre de la nouvelle génération, auteur raté parce que trop lucide, amant malheureux, qui répète : «Il nous faut des formes nouvelles!» Pour toutes sortes de raisons, mauvais caractère, conflit avec son maître Nemirovič-Dančenko, Meyerhold est poussé hors du théâtre lors de sa réorganisation en 1902.

Il forme une compagnie avec de jeunes acteurs du Théâtre artistique et loue une salle dans la ville de Kherson, gros bourg au centre d'une région céréalière très riche en Ukraine. Les notables locaux tiennent à leur théâtre et sont flattés d'avoir des acteurs formés à Moscou par le Théâtre artistique. La «Troupe d'artistes dramatiques russes », comme elle s'appelle, impose d'emblée sa marque en ouvrant la saison par Les Trois sœurs (Tri sestry). Elle alterne des œuvres classiques (Ostrovskij) et celles de dramaturges contemporains, comme Nemirovič, Zuderman, Heijermans, Hauptmann, Ibsen ou Lev Tolstoj. Cela dit, comme pour 
tester son public, la troupe présente dès le mois d'octobre une première œuvre symboliste, La Toison d'or, du Polonais Przybyszewski, en présence de l'auteur. Ce drame en 3 actes, dont le titre symbolise la quête à tout prix du bonheur, présente une légitimation de l'adultère. L'auteur explique :

Le drame est celui des sentiments et des pressentiments, des remords, de la lutte intérieure, c'est le drame de l'inquiétude, de l'horreur et de l'épouvante ${ }^{4}$.

La représentation déroute d'autant plus le public qu'elle se déroule presque entièrement dans l'obscurité.

C'est à Sébastopol, où elle effectue une tournée au printemps 1903, que la troupe ose enfin proposer au public un échantillon d'œuvres relevant de la sensibilité nouvelle. Sous le nom de « Soirée d'art nouveau », on donne tout un récital poétique : deux pièces, l'une de Schnitzler, Les derniers masques, l'autre de Maeterlinck, L'Intruse, ensuite des tableaux vivants, enfin des récitations poétiques.

Un critique constate que « les tableaux vivants, réalisés avec soin et intelligence, ont apparemment plu au public. Pris par l'interprétation parfaite des vers d'Edgar Poe et de Bal'mont (par MM. Meyerhold et Pevcov), on en a oublié l'heure si bien qu'on a terminé après minuit ! »5

L'accompagnement musical était composé de pièces de Grieg. Quant à l'éventail des poèmes récités, il est caractéristique : Baudelaire, Verlaine, Edgar Poe, Maeterlinck et Bal'mont.

L'artiste Kostin, spécialiste des tableaux vivants, représente quatre œuvres de Böcklin et une de Vrubel' $^{\prime}$, le tableau Le Démon et Tamara, illustration du poème de Lermontov (datant des années 1890). La volonté de synthèse des arts est donc à l'œuvre, avec les moyens propres au spectacle même si on peut se demander s'il n'y a pas quelque chose de frelaté dans cette tentative de vulgarisation.

On sait que l'intruse qui donne son nom à la pièce de Maeterlinck est la mort, à l'œuvre sans bruit dans le calme d'une famille dont la mère mourra à la fin de la pièce. Qu'en dit le critique ?

L'Intruse de Maeterlinck appartient aux œuvres symbolistes qui doivent susciter chez le spectateur l'atmosphère voulue par l'auteur, mais le théâtre n'y réussit pas toujours, laissant souvent la place à l'ennuí'.

Un détail de mise en scène : pour Maeterlinck, sont assis autour de la table les six membres de la famille (grand-père aveugle, père, oncle et trois filles). Dans ses notes Meyerhold indique seulement «quatre chaises ». Au moment où le grandpère croit voir un personnage, en face de lui, une des chaises restera vide, les deux autres étant occupées par le père et l'oncle. Tout est là. Comment faire sentir la

4. S. Przybyszewski, O drame i scene, in Sočinenija (Euvres) (en russe), Moscou, 1910, t. 4, p. 340 .

5. S. Spiro, Krymskij vestnik, 20 mai 1903.

6. Ibid. 
présence de choses ou de personnes invisibles ? Il semble malheureusement que les jeunes acteurs de la troupe n'étaient pas toujours capables d'incarner le rêve du metteur en scène.

Lors de la seconde saison en province (1903-1904), la troupe devient la «Compagnie du nouveau drame». Meyerhold annonce d'emblée la couleur. Il augmente la part des œuvres « modernistes », Ibsen, Schnitzler, Hauptman, tout en reprenant à l'identique le répertoire du Théâtre artistique, Ostrovskij (La princesse des neiges - Sneguročka) Shakespeare (La nuit des rois), et même Gor'kij (Les bas-fonds - Na dne). Il choisira également une pièce de Maeterlinck, mais qui n'appartient pas à proprement parler au répertoire symboliste. Il s'agit de Monna Vanna où le dramaturge renoue avec la tradition éprouvée du théâtre historique.

Le défi au public est lancé avec une nouvelle œuvre de Przybyszewski, Neige. Comprenant que, pour ces œuvres symbolistes, il faut jouer de toute la palette des moyens à sa disposition, Meyerhold travaille les éclairages grâce auxquels il pense pouvoir révéler le plan de l'éternité, tandis que les personnages doivent représenter des entités métaphysiques. On y assiste à la marche inexorable du destin au sein d'une réalité paisible, immobile et même banale. Le drame se déroule au plus profond de l'hiver, un hiver recouvert d'une épaisse couche de neige. La neige est pure et blanche comme la mort. Le héros vit heureux avec sa femme Bronka, mais Eva, la femme fatidique, sera la cause de leur malheur. Emporté par une force irrésistible, Thaddée s'en va avec Eva, tandis que Bronka, abandonnée, se jette dans une crevasse du lac gelé. On ne retrouvera son corps qu'au printemps. Le public siffle la pièce sans retenue.

Aleksej Remizov, qui est à l'époque conseiller littéraire de Meyerhold, défend ses amis :

Le grand sens artistique du metteur en scène s'est manifesté dans la réalisation scénique, qui a su combiner la musique, les couleurs et la plastique propres au symbolisme du drame et à son déroulement réel, avec, de la part des acteurs, l'attitude voulue, toute d'amour pour leur rôle. [...] Eva n'entraîne pas aveuglément l'homme au milieu des cadavres, au milieu des victimes de ses crimes, allant toujours droit devant soi. [...] Elle ne l'entraîne pas par la musique lilasfoncé de ses refrains : «Il faut d'abord dompter les mers, creuser les montagnes, connaître tous les tourments et toutes les jouissances pour ouvrir les yeux sur le monde nouveau, et si par hasard le conquistador écrase sous son pas de fer une fleurette, que peut-on y faire ? Que peut-on y faire? » Le public interloqué faisait semblant de comprendre, ou bien fronçait bêtement son front bas, affreux. Après cela ce qu'il a pu rire ! : « Il faut d'abord dompter les mers ! Ha ! Ha ! »7.

Remizov résume en quelques mots la philosophie des compagnons du nouveau drame :

Le théâtre n'est pas un amusement, un divertissement, le théâtre n'est pas la copie de la faiblesse humaine, c'est un culte, une liturgie qui recèle peut-être

7. Aleksej Remizov, « Tovariščestvo novoj dramy » (La Compagnie du nouveau drame), Vesy, 4, 1904, p. 36. 
dans ses mystères la Rédemption. [...] Voilà le théâtre dont rêve le Nouveau Drame ${ }^{8}$.

Ayant épuisé les charmes de la ville de Kherson, Meyerhold obtient pour la nouvelle saison (1904-1905) un contrat à Tiflis, capitale de la Géorgie, l'Athènes du Caucase. Malgré le niveau culturel élevé de son public, Tiflis ne fait pas meilleur accueil aux deux œuvres phares du style moderne, La Toison d'or et Neige, qui sont toutes les deux abondamment sifflées.

\section{III}

Pendant que le disciple engage le fer pour initier à la sensibilité nouvelle le public provincial, son maître Stanislavskij cherche, lui aussi, à traduire dans le langage de la scène des œuvres qui font appel au mystère. Le modernisme est dans l'air, il est diffusé par les grandes revues à la mode, comme Le Monde de l'art (Mir iskusstva). Stanislavskij s'intéresse donc à trois pièces de Maeterlinck : Intérieur, l'Intruse et Les aveugles où l'auteur met en œuvre son esthétique du «théâtre immobile ». C'est d'ailleurs Čehov qui a conseillé au metteur en scène de monter ces pièces qui viennent d'être traduites en russe. Il suggère de les jouer sur un fond musical. Mais, de plus en plus affaibli, l'ami du Théâtre artistique, part se soigner en Allemagne où il meurt à Badenweiler sans avoir pu assister à la création du spectacle Maeterlinck.

Stanislavskij va s'efforcer de trouver la clef d'une œuvre qui constitue une étape décisive vers l'abandon du théâtre de mœurs, du théâtre de représentation de la vie extérieure. Et puis le metteur en scène, qui est en train de réfléchir à son système de formation de l'acteur et qui est conscient de l'importance du courant souterrain qui sous-tend tout dialogue, ne peut pas ne pas être sensible à ce qu'en dit le dramaturge lui-même. C'est chez Ibsen que Maeterlinck repère les germes de ce dialogue caché :

Qu'est-ce que le poète a ajouté à la vie pour qu'elle nous apparaisse si étrange, si profonde et si inquiétante sous sa puérilité extérieure ? [...] Hilde et Solness sont, je pense, les premiers héros qui se sentent vivre un instant dans l'atmosphère de l'âme, et cette vie essentielle qu'ils ont découverte en eux, par delà leur vie ordinaire, les épouvante 9 .

On peut se demander si Stanislavskij n'a pas été lui-même épouvanté par cette totale remise en cause des normes scéniques. Pour ne prendre que Les Aveugles, il s'insurge contre le règne absolu de la mort que cela implique. C'est ce qu'a bien perçu un critique :

Il est significatif que le Théâtre [artistique] a fait passer la pièce du mode mineur au mode majeur. Il y a chez Maeterlinck une angoisse et une tristesse indépassa-

9. Maurice Maeterlinck, Le Trésor des humbles, Bruxelles, Labor, 1986, p. 109. 
bles. [Pour lui] la seule vérité de la vie est dans la mort. Au Théâtre artistique, la pièce est presque devenue un hymne à la lumière, un élan hardi vers l'avenir, un avenir prometteur, annonçant la victoire sur les ténèbres, surtout à la fin. La jeunesse vaincra la cécité, dissipera les ténèbres, anéantira la mort. C'est ce qu'on sentait dans les intonations, dans les infléchissements du texte, texte qui par son caractère ondoyant et obscur laisse de grandes possibilités d'interprétation ; c'est ce que suggérait la gestuelle. L'accent était mis non sur la mort, mais sur la cécité comme allégorie. Le prêtre mort que Maeterlinck place à la tête des aveugles était relégué dans un coin ; il n'était presque jamais au centre de la pièce ; on l'oubliait lors de l'accord final ${ }^{10}$.

Le metteur en scène avoue franchement la difficulté qu'il a eu à pénétrer le sens de cette pièce :

Au début on a eu du mal avec Maeterlinck. La première fois où nous avons fait une lecture des Aveugles, on a ri aux larmes. Au début, on a pris un ton théâtral, c'était ridicule, après on y a ajouté des nuances de caractère réaliste, ce qui était encore plus ridicule ; et puis on a enfin trouvé le ton actuel quelque peu solennel, mystérieux et angoissant. [...] À cause du public, on a été obligé de transiger. L'horreur de l'existence aveugle aurait été bien plus perceptible si les comédiens étaient restés immobiles, attentifs à leurs mouvements intérieurs, sans gestes, sans mimique, insensibles comme des pierres, mais cela ne peut se faire que dans une petite salle, pour un public restreint, spécialement choisi. Dans notre théâtre cela aurait suscité l'ennui, et puis on n'aurait pas manqué d'entendre cette toux qui résonne toujours au théâtre, glace les artistes et relâche leur attention ${ }^{11}$.

Stanislavskij est donc pleinement conscient de l'écart qu'il y a entre le projet et la réalisation. Il souffrira de ce qu'il ressent comme un échec, mais son sens artistique reste en éveil.

Comment pourrait-on rendre par [des] formes vulgaires la vie spirituelle de l'homme, ce qu'elle a de supraconscient, de noble, d'élevé, ce qui fait la profondeur et la beauté d'un Vroubel, d'un Maeterlinck, d'un Ibsen ?12

La méthode de jeu mise au point au Théâtre artistique, et qui fait sa force, ne seraitelle donc pas universelle?

Stanislavskij a entendu parler des tentatives de Meyerhold en province. La troupe du transfuge n'était-elle pas une sorte de filiale du Théâtre artistique ?

10. F. «Spektakl` Meterlinka ( (Le spectacle de Maeterlinck), Novosti dnja, 3 octobre 1904.

11. «Stanislavskij i svoboda tvorčestva » (Stanislavskij et la liberté de création), interview, Teatral'naja Rossija, 18, 1905, p. 308.

12. Constantin S. Stanislavski, Ma Vie dans l'art, trad. Denise Yoccoz, Lausanne, L'Âge d'Homme, 1980, p. 353. 


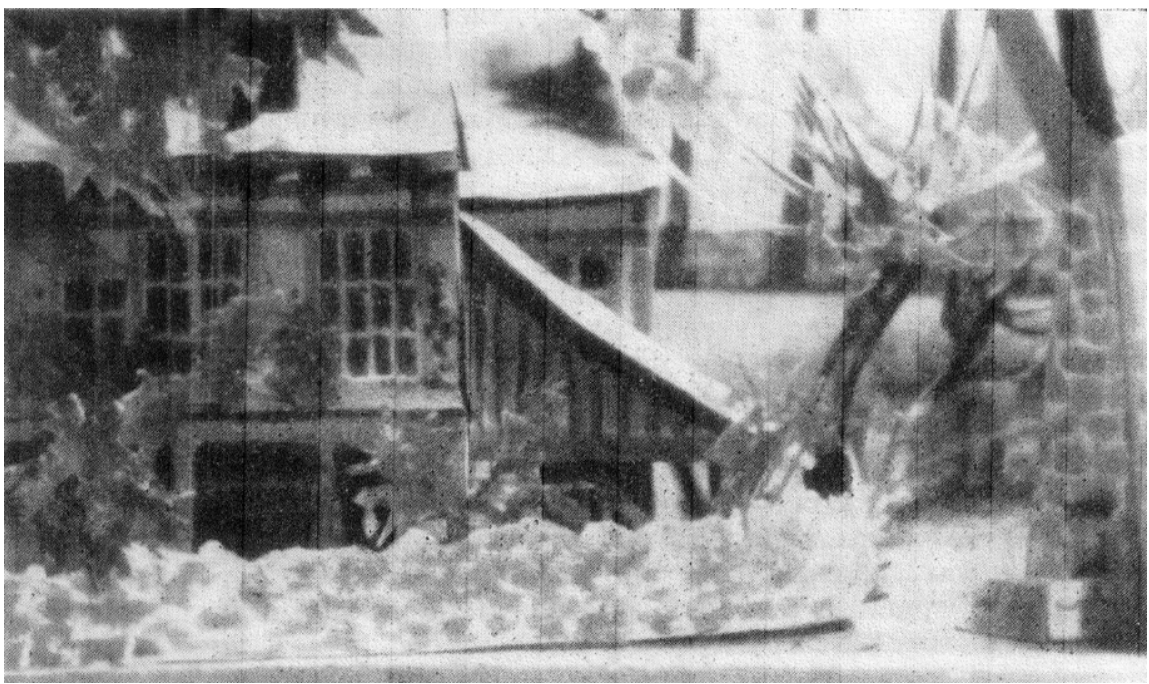

Vardkes Surenianc

Maquette du décor de la pièce de Maeterlinck Intérieur au Théâtre artistique (1904)

Tandis que je ne faisais qu'aspirer au nouveau, sans avoir trouvé encore le chemin ni le moyen d'y parvenir, Meyerhold, apparemment, avait déjà trouvé de nouvelles voies et de nouveaux procédés. S'il ne pouvait les mettre pleinement en pratique cela tenait d'un côté à des problèmes matériels, de l'autre au niveau assez bas de sa troupe. J'avais donc en Meyerhold l'homme qui m'était nécessaire dans la période de recherches où je me trouvais. Je décidai de l'aider à poursuivre ses travaux qui, me semblait-il, avaient beaucoup en commun avec mes propres rêves ${ }^{13}$.

Et c'est l'aventure du Théâtre-Studio qui durera six mois (mai à octobre 1905). Conscient de la difficulté qu'il y a à proposer une vision nouvelle du répertoire et du jeu de l'acteur au public du Théâtre artistique, Stanislavskij s'embarque dans une aventure incroyable : il loue un théâtre de jauge moyenne dont il fera une scène expérimentale. D'où le nom de Théâtre-Studio qu'il lui donne. Il laisse carte blanche à Meyerhold auquel il fait entièrement confiance. Celui-ci porte son choix sur des œuvres de dramaturges contemporains, connus pour leur modernisme : Hauptmann, Schnitzler, Przybyszewski, Ibsen et Maeterlinck. Il aborde en premier lieu ce «drame pour marionnettes » qu'est La Mort de Tintagiles, situant d'emblée le niveau de son ambition de metteur en scène novateur.

Chacun des deux organisateurs expose son credo. Pour Meyerhold :

Les formes contemporaines de l'art dramatique se survivent à elles-mêmes depuis longtemps. Le spectateur contemporain exige d'autres procédés techniques. Le Théâtre artistique est parvenu à la virtuosité sur le plan du naturalisme 
et de la simplicité naturelle de l'interprétation. Mais des drames sont apparus qui exigent de nouvelles techniques de mise en scène et d'interprétation ${ }^{14}$.

Quant à Stanislavskij, il n'est pas moins enthousiaste :

Le credo du nouveau Studio [peut] se résumer en quelques mots : le réalisme, la couleur locale ont vécu. Le temps est venu de porter sur scène l'irréel. Ce n'est pas la vie telle qu'elle est, telle qu'elle s'écoule, que la scène doit peindre mais la vie dont nous avons confusément l'intuition dans nos songes, dans nos visions, dans nos moments d'exaltation. Voilà l'état d'âme qu'il faut traduire scéniquement, exactement comme le font les peintres de la nouvelle école sur leurs toiles, les musiciens modernes dans leur musique, les nouveaux poètes dans leur poésie. [...] La force de l'art nouveau réside dans les combinaisons, les harmonies, les correspondances de couleurs, de lignes, de notes et de mots. Elles créent un état d'esprit général dont le spectateur s'imprègne inconsciemment ${ }^{15}$.

Si Meyerhold est plus polémique (le nouveau théâtre contre le théâtre « naturaliste ») les deux hommes ont en commun le souci de faire pénétrer le théâtre dans le monde de la poésie, de le sortir du simple divertissement pour le hausser au rang d'un des beaux-arts.

Quelle vision Meyerhold a-t-il de La Mort de Tintagiles ?

Il confie à Sudejkin le soin de réaliser des esquisses éthérées à partir des croquis qu'il lui donne. Quant aux mouvements des acteurs, ils sont dessinés dans des schémas très précis, accompagnés de commentaires détaillés.

Voici les règles qu'il pose :

Maeterlinck construit le dialogue «extérieurement nécessaire » de telle façon que les personnages doivent prononcer un minimum de mots pour une tension maximale de l'action.

Pour rendre manifeste aux spectateurs ce dialogue « intérieur » des drames de Maeterlinck, pour les aider à le percevoir, les gens de théâtre doivent chercher de nouveaux moyens d'expression ${ }^{16}$.

Le jeu est constitué d'une succession d'attitudes plastiques séparées par des pauses.

Là, ils s'arrêtent. Tintagiles abaisse les bras devant la balustrade (une fleur à la main et cela tout au long de l'acte). Ygraine s'arrête et le contemple. Une pause. Elle se met alors à parler. [...] Ygraine descend en silence l'escalier qui conduit à la tonnelle. Faisant face à la banquette, le dos au public, elle tourne son épaule droite et s'assied à l'extrémité du côté droit. La tête baissée, les mains jointes posées sur les genoux, elle se met à parler. [...] Même pose, mais la tête levée. [...] Nouveau bas-relief. Elle porte les mains à son visage (madone)... ${ }^{17}$.

14. Vs. Meyerhold, Écrits..., op. cit, vol. 1, p. 89.

15. C. Stanislavski, op. cit., p. 357.

16. Vs. Meyerhold, Écrits..., op. cit., vol. 1, p. 107.

17. Vs. Meyerhold, Cahiers de régie (La Mort de Tintagiles). Rossijskij gosudarstvennyj arhiv literatury i iskusstva (RGALI - Archives littéraires et artistiques de Russie), f. 998, op. 1, d. 196,1. 1 . 
Meyerhold élabore les règles permettant de traduire dans le corps de l'acteur les modes d'expression adaptés à l'écriture nouvelle. Ces règles seront définies avec précision dans le domaine de la diction, avec plus d'hésitation en ce qui concerne la plastique dans la mesure où celle-ci doit s'harmoniser avec le travail du scénographe :

A - La diction :

- Une froide ciselure des mots. Pas de trémolos.

- Le son doit toujours avoir un support, les mots doivent tomber comme des gouttes d'eau dans un puits profond.

- Frémissement mystique plus puissant que le tempérament du vieux théâtre.

- Les émotions de l'âme et leur tragique sont liés indissolublement à l'émotion issue de la forme.

- Il faut éviter à tout prix le débit rapide qui ne se conçoit que dans les drames « neurasthéniques ».

- Le tragique avec le sourire aux lèvres. Savonarole : «Au pied de la croix [la Vierge] se tenait triste et heureuse, pénétrée du secret de l'immense bonté divine ».

L'acteur nouveau sait exprimer le degré supérieur du tragique de la même façon. [...] un calme extérieur presque froid, sans cris ni pleurs, sans trémolos, mais avec un grande profondeur ${ }^{18}$.

B - La plastique

- Wagner laisse à l'orchestre le soin de parler des sentiments, de même je confie ce rôle aux mouvements plastiques.

- Les tableaux de Maeterlinck sont archaïsés. [...] L'objectif [est] de concentrer sur les mouvements toute l'attention des spectateurs. [...] Ce qui explique le choix d'un fond décoratif unique pour La Mort de Tintagiles ${ }^{19}$.

Certes les observations de Meyerhold ont été écrites sept ans après l'événement, mais c'est pour lui une expérience majeure qui l'a marqué.

L'influence du Théâtre-Studio sur les destinées du théâtre russe fut importante ; on peut en juger par le fait qu'après sa disparition, toutes les fois qu'à Moscou ou à Saint-Pétersbourg on montait un spectacle remarquable, c'est toujours à ce même Théâtre-Studio qu'il faisait penser ${ }^{20}$.

Cet enfant mort-né restera par la suite l'enfant chéri de Meyerhold. Stanislavskij vient assister à la répétition générale de La Mort de Tintagiles. Il est horrifié. De mauvais acteurs psalmodient dans une semi-obscurité. C'est intolérable. «Au théâtre, il faut voir le visage des comédiens ! » Il réclame de la lumière. Mais tout l'effet de mystère disparaît alors. Profitant des événements révolutionnaires d'octobre 1905 qui paralysent toute activité, Stanislavskij met fin à cette expérience.

18. Vs. Meyerhold, Écrits..., op. cit, vol. 1, p. 114-115.

19. Ibid., p. 116-117.

20. Ibid., p. 93-94. 
Après la fermeture du Studio, Meyerhold reprend ses fonctions de directeur de la Compagnie du nouveau drame. Ce qu'il n'a pu faire à Moscou, il le fera dans la capitale du Caucase. Conscient du caractère déconcertant de cette œuvre, il en fait l'exégèse. Dans une envolée prophétique, il opère un rapprochement entre la révolte métaphysique contre le destin et la révolte sociale des hommes épris de justice contre une société inhumaine. (En filigrane, il y a là toute la polémique future entre Camus et Sartre).

\begin{abstract}
Essayez, lorsque vous écouterez la pièce de vous révolter avec Ygraine, non pas contre la mort mais contre ce qui donne la mort. La signification symbolique de la pièce atteindra alors à des sommets vertigineux ; si l'on s'indigne, ce n'est pas contre la Mort, mais contre ce qui donne la mort. Alors on comprendra que l'île où se passe l'action, c'est notre vie, et que ce château qui se cache derrière des arbres mornes et desséchés, ce château est une prison. Tintagiles, c'est la jeunesse confiante, belle, idéale et pure. Et il y a Quelqu'un qui met impitoyablement à mort ces êtres jeunes et magnifiques. Sœurs, mères et enfants tendent vers le ciel leurs bras impuissants ; ils implorent la grâce, le pardon, la clémence, la liberté. Sur notre île, gémissent et meurent des milliers de jeunes et beaux Tintagiles ${ }^{21}$.
\end{abstract}

Peut-être faut-il voir là la force et la faiblesse de Meyerhold ? Il est capable de toutes les compromissions au nom de sa conception du théâtre. Faire passer une pièce symboliste pour une œuvre de combat social. Plus tard adhérer au parti communiste de Russie parce que c'est la condition sine qua non de la survie du théâtre tel qu'il le conçoit, et cela malgré toutes les réserves qu'il formule intérieurement à l'égard des « insurgés » de novembre 1917.

Son tempérament d'artiste reprend le dessus lorsqu'il demande au public de se comporter devant cette pièce non comme devant une œuvre faite pour être vue (spectateur), mais écoutée : « lorsque vous écouterez la pièce... ».

Le plus grand de tous les arts est la musique. Le plus grand. Tous nous en avons besoin. Â tous elle est chère sans exception. Tout le monde aime la musique, tout le monde ne la comprend pas. Ceux qui ne la comprennent pas ne cherchent pas à en expliquer l'existence ni la signification. Et en même temps chacun cherche au fond de soi-même une explication. Le sens de toute musique, sans exception, devient clair quand l'âme de l'auditeur se fond avec celle du compositeur ${ }^{22}$.

21. Vs. Meyerhold, «Mot d'introduction avant la première de la Mort de Tintagiles à Tiflis », in Écrits..., op. cit., vol. 1, p. 80.

22. Vs. Meyerhold, Écrits..., op.cit., vol. 1, p. 79. 
La tristesse évoquée par le compositeur d'une marche funèbre à l'égard d'un événement donné va être intériorisée par les auditeurs. L'un se remémorera une douleur personnelle, l'autre éprouvera de la compassion pour les victimes des injustices.

La Mort de Tintagiles, c'est aussi une musique. Mille spectateurs. Mille interprétations, si tant est qu'il faille donner une interprétation à la musique.

En voici la plus simple : vous allez voir la mort d'un garçon à la fleur de l'âge, et le chagrin de sa sœur qui n'a pu le sauver ni par des supplications, ni par d'humbles prières, ni par des menaces, ni par des flatteries. La Reine dont il est tant question dans la pièce, c'est la mort. Voilà l'explication la plus simple ${ }^{23}$.

Le metteur en scène est bien conscient qu'une œuvre symboliste se déploie sur plusieurs dimensions de la conscience et notamment la dimension musicale. On doit ressentir une expérience à la fois esthétique et existentielle. Le théâtre n'est ni une tribune ni une chaire. Mais craignant une réaction impropre du public, Meyerhold lui donne une sorte de mot de passe qui, par analogie, devrait permettre de saisir le mystère contenu dans La Mort de Tintagiles.

En 1906-1907, le dialogue Meyerhold-Maeterlinck est à son plus haut point d'intensité à Saint-Pétersbourg. Komissarževskaja l'avait fait venir pour faire du Théâtre dramatique le porte-drapeau du symbolisme. Meyerhold se lance avec frénésie dans cette aventure. Il donne un faisceau d'œuvres de Maeterlinck : Sœur Béatrice, avec son aspect religieux et moyenâgeux ; Le Miracle de Saint Antoine où le symbolisme sert d'arme contre le monde «bourgeois », enfin Pelleas et Mélisande, qui traduit l'aspect irréel, incompréhensible de l'existence.

Il s'adresse aussi à Przybyszewski dont il donne Le Conte éternel et enfin au poète russe Aleksandr Blok, dont il monte la pièce décapante La Baraque de foire (Balagančik). Cette pièce en un acte apparaîtra en fait comme un adieu au symbolisme, un adieu à l'irréalité, un adieu déchirant et ironique à la fois.

Lorsque Meyerhold monte, à l'automne 1906, Sœur Béatrice, le spectacle est considéré comme d'une beauté exceptionnelle. Tout le monde en salue la réussite, d'autant plus qu'il permet à Komissarževskaja, dans le double rôle de la religieuse pécheresse et de la Sainte Vierge, de réaliser son vœu d'un rajeunissement du théâtre par le biais du symbolisme. La référence à Vrubel' qu'on trouve sous la plume de Meyerhold est symptomatique :

Du côté opposé apparaissaient trois jeunes pèlerins, appuyés sur de longs bâtons filiformes, vêtus de bure (avec des visages «à la Vroubel »); ils s'agenouillaient, les mains levées au-dessus de leur tête ${ }^{24}$. 


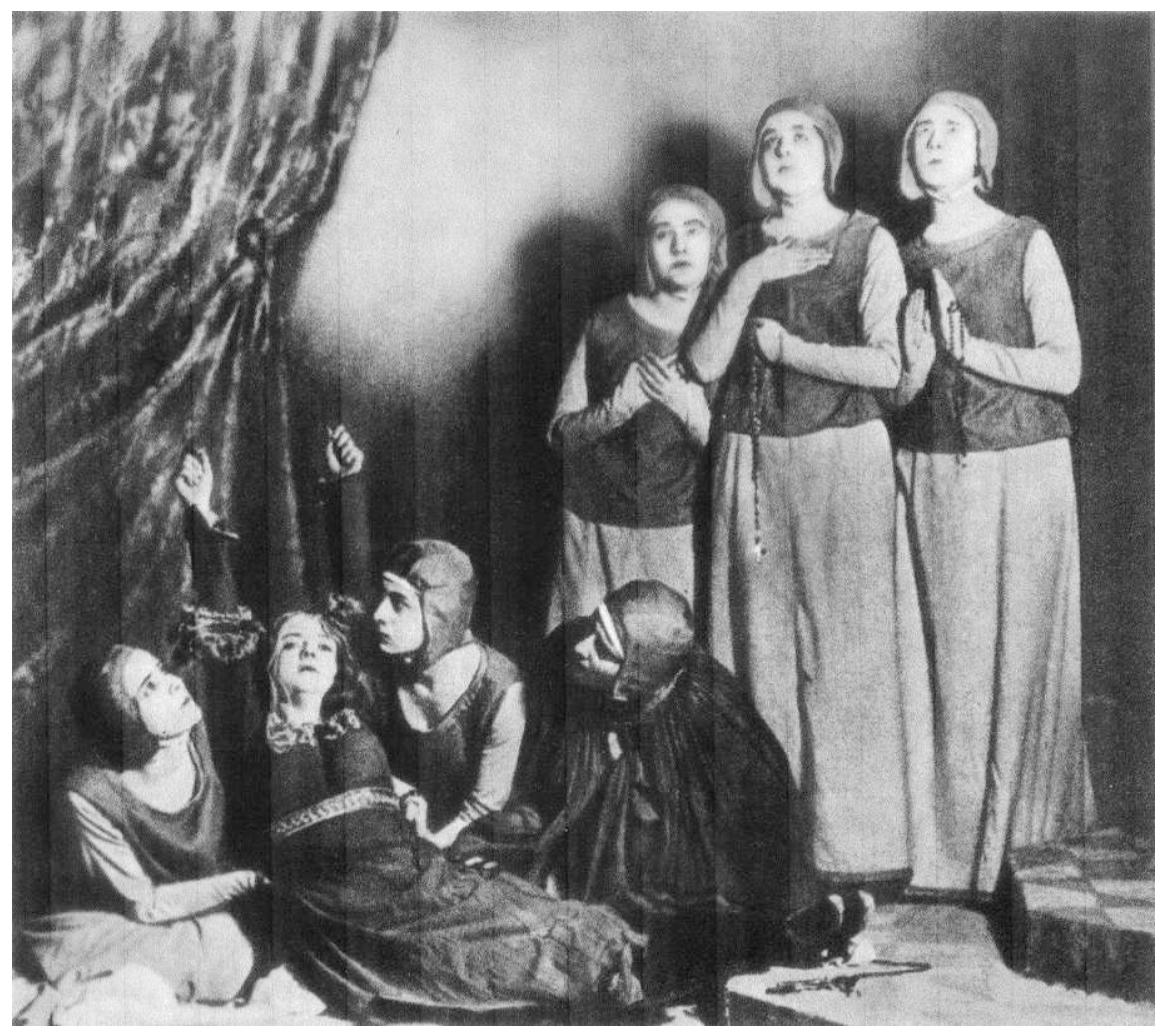

Scène de groupe de la pièce de Maeterlinck,

Sœur Béatrice, au théâtre de Komissarževskaja (1906)

Le style du peintre symboliste s'est tellement imposé à la conscience collective qu'il devient une référence banale. Il est curieux de constater que Meyerhold se réfère de nouveau au peintre en 1936, pour revendiquer la liberté de création, alors qu'il est attaqué par ses autorités de tutelle :

Prenez Serov et Vroubel. Serov était un homme sain et Vroubel était malade. Tous deux ont donné des œuvres remarquables. Mais s'il avait été en excellente santé, Vroubel nous aurait donné des œuvres encore plus remarquables. En art, il arrive souvent qu'une expérience saine inclue du pathologique; c'est un problème pour les historiens de l'art. Nous devons aussi savoir ce qui, dans une expérimentation saine, est utile, et ce qui doit être rejeté. Le Comité pour les affaires artistiques doit se poser le problème de l'expérimentation. [...] Il doit aussi penser à ne pas jeter le bébé avec l'eau du bain ${ }^{25}$.

C'est à ce moment-là que se déclenche toute une polémique relative à la marionnette. L'acteur symboliste ne devrait-il pas tout simplement être remplacé par une marionnette? Maeterlinck n'a-t-il pas écrit ses drames pour «théâtre de 
marionnettes » ? Il y a une raison très simple à cela, c'est que les germes du théâtre symboliste ont été semés par le Petit théâtre de marionnettes qui a fonctionné à Paris de 1888 à 1893. On y montait des œuvres de grande envergure : Les Mystères d'Eleusis, Le Mystère de la Nativité de Noël, des adaptations d'Aristophane, de Cervantès, Le Chariot de terre cuite de Kalidasa, La Tempête de Shakespeare... Tous les ingrédients du modernisme y sont présents : théâtre antique, mystères sacrés, les grands classiques et les traditions extra-européennes. Pourquoi des marionnettes ? Parce qu'on pouvait obtenir ainsi un caractère aérien, une théâtralité pure que ne pouvait donner le théâtre avec acteurs. Est-ce à ce théâtre que pensait Maeterlinck ? Sans doute. Il aurait été surpris si on lui avait dit qu'on allait demander à des acteurs en chair et en os d'imiter des marionnettes.

Le public s'interroge : l'acteur doit-il prendre la marionnette comme modèle ? Le metteur en scène a-t-il le droit d'exiger de ses comédiens de se comporter comme des marionnettes ?

L'un des protagonistes de ce débat est l'Anglais Gordon Craig qui, dans son livre On the art of the theater, affirme que l'acteur doit précisément calquer son jeu sur celui des marionnettes et devenir une « supermarionnette ». Cette revendication met le feu aux poudres et on accuse Meyerhold d'en agir ainsi avec ses acteurs. Les critiques l'accusent de transformer Komissarževskaja, la vedette éthérée, qui séduit par son charme et son tempérament, en une «marionnette » à deux dimensions. Ébranlée par ces critiques, elle rompra brutalement le contrat qui la liait à Meyerhold. Le coup de grâce fut donné par Andrej Belyj, un des coryphées du symbolisme qui affirmait perfidement :

M. Meyerhold fait-il des pas en direction du théâtre de marionnettes ? Oui, et c'est un mérite indéniable de ce théâtre. [...] Voilà donc la voie véritable du théâtre de Komissarževskaja, sauf que l'actrice n'a rien à faire dans ce théâtre. Son talent serait détruit et ce serait regrettable ${ }^{26}$.

Autre polémique, celle concernant la signification du symbolisme en Russie. La Baraque de foire présente des personnages de la commedia dell'arte égarés dans les rues de Saint-Pétersbourg et pénètre dans les méandres des rêveries du poète. Le metteur en scène a su mobiliser tous les moyens qu'offre la machine théâtrale pour les mettre au service de la poétique symboliste: petit théâtre construit sur le plateau, paysage peint que l'on déchire pour en dénoncer l'illusion, utilisation de l'avant-scène pour prendre à partie le public.

Autant le praticien a trouvé le style qui convenait à cette œuvre symboliste, autant le théoricien du théâtre qu'était Meyerhold semble étranger à la conception du monde véhiculée par le symbolisme. On se demande s'il ne s'est pas contenté de se prêter au jeu le temps nécessaire à la mise au point de moyens d'expression qui lui serviront plus tard pour un autre répertoire. La seule pièce russe montée par lui lors de son pèlerinage en « symbolie » est cette satire grinçante dans laquelle Blok

26. A. Belyj, «Simvoličeskij teatr. Po povodu gastrolej Komissarževskoj » (Le théâtre symboliste. À propos de la tournée du théâtre de Komissarževskaja), Utro Rossii, 16 septembre 1907. 
sonne le glas du symbolisme en s'adonnant à un exercice d'auto-dénigrement féroce. Le poète dénonce avec une ironie décapante les poncifs symbolistes sans s'épargner lui-même. Tout y passe, l'attente de l'Éternel féminin, la passion démoniaque de l'amour tsigane, l'héroïsme sublime du chevalier du Moyen-Âge, le sacré émanant des voûtes gothiques, et tout y sombre dans son contraire, sous les coups d'un Arlequin bousculant Colombine et ridiculisant Pierrot. La pièce s'ouvre par la parodie d'une scène mystique où les adeptes de la religion de la mort sont ridiculisés par Pierrot et Colombine. Mais non, s'écrie celui-ci, «ce n'est pas la mort, c'est ma fiancée! ». Alors ces mystiques, caricatures des symbolistes, sont physiquement anéantis, transformés en figures de carton-pâte.

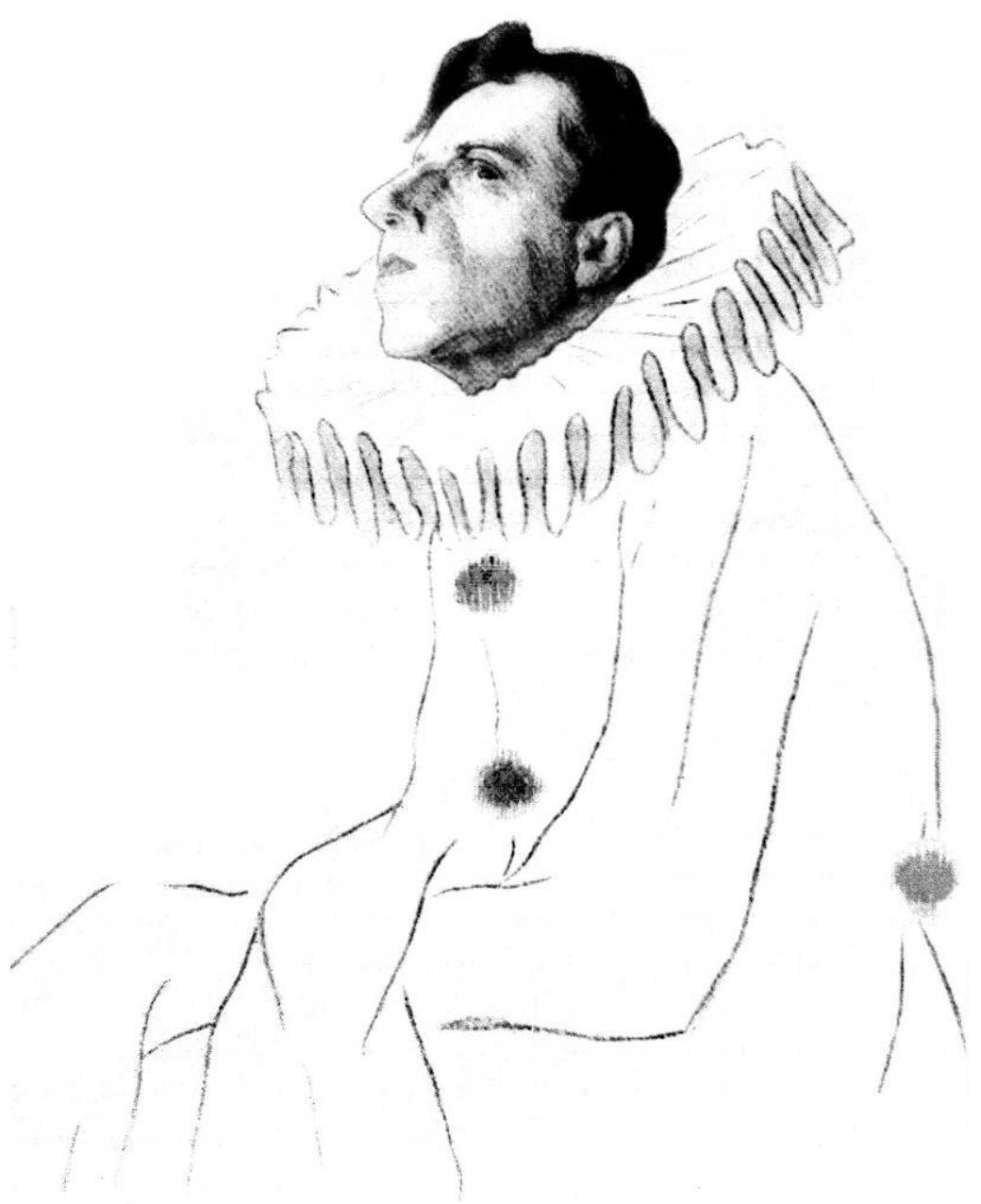

Nikolaj Uljanov. Portrait de Vsevolod Meyerhold dans le rôle de Pierrot dans le spectacle La Baraque de foire d'Aleksandr Blok au théâtre de Kommissarževskaja (1906) 
Un détail qui a choqué les contemporains, c'est le cri d'un paillasse blessé affirmant qu'il saigne du « jus de groseille ». On y voit un blasphème contre la mission sacrée du poète qui verse son sang comme le Christ pour la multitude !

Pierrot, joué par Meyerhold, vient avec naïveté comme au sortir d'une haute maladie clore le mystère à l'envers en jouant du pipeau face au public (du pipeau et non du luth) à qui il reproche de ne voir en lui qu'un pantin, de se moquer de sa souffrance. Comme si Blok - et avec lui Meyerhold - voulait se débarrasser des colifichets symbolistes pour retrouver la vérité de la poésie et de la vie saine de l'esprit.

La sincérité de Meyerhold en tant qu'artiste ne peut être mise en doute lorsqu'on lit ces quelques lignes griffonnées à l'intention d'une de ses admiratrices :

Mon art est la flamme qui m'aide à ne pas remarquer les épines du chemin. En dehors de cet art je ne suis rien. [...] Comme vous, $\mathrm{j}$ ' aspire à gagner les hauteurs. Je suis en recherche. J'ignore les mystères de la vie et ne sais pas si je parviendrai un jour à les connaître 27 .

Le symbolisme a secoué la machine théâtrale en la contraignant à jouer de tous ses muscles encore inutilisés: le texte ne devenait qu'un des éléments d'une symphonie où jouaient des instruments aussi divers que l'acteur avec son corps, sa diction, son costume, son agilité, le scénographe avec ses constructions inhabituelles. Le symbolisme au théâtre réclamait par sa poétique même la présence d'un homme chef d'orchestre capable d'imposer une vision cohérente et d'harmoniser tous ces éléments conflictuels. Le symbolisme allait parfaire le travail commencé à son insu par Čehov et sceller le destin du metteur en scène, exégète et créateur du spectacle. Ainsi, partant d'une réalité fluide et poétique, le symbolisme se nie en tant que tel pour donner naissance à un art en quête d'objectivité.

Ce qui en a fait la grandeur c'est qu'il a osé regarder droit dans les yeux la souffrance humaine qu'il pensait pouvoir adoucir. Les grands noms du symbolisme russe, Vrubel' pour la peinture, Skrjabin pour la musique, Blok pour la poésie, ont tous été des êtres familiers de la souffrance. Ils ont cherché à surmonter la douleur d'être en mobilisant les formes sensibles par un jeu de combinaison, peinture et poésie pour l'un, musique et couleurs pour l'autre, poésie, musique et couleurs pour le troisième.

En ce sens Meyerhold a bien participé à la tentative symboliste visant à exprimer sous une forme sensible l'inexprimé, cet inexprimé que chaque être porte dans son inconscient et qui est le réceptacle de sa vérité et la condition de son salut.

gerard.abensour@infonie.fr

27. Vs. Mejerhol’d, « Pis'mo zritel'nice » (Lettre à une spectatrice), printemps 1904, in Mejerhol'd i drugie (Meyerhold et les autres), Moscou, 2000, p. 52. 